Conclusion While the presence of infected necrosis or persistent organ failure in SAP (group III) is associated with high mortality, the combination of "infected necrosis and persistent organ failure" (group IV) is uniformly fatal. Further research is necessary to confirm the findings in our study and to explore ways of optimising patients in group III to improve survival.

Competing interests None declared.

\section{PWE-136 PRE-OPERATIVE ERCP, CHOLECYSTITIS AND MALE GENDER ARE THE MAJOR PREDICTORS OF DIFFICULT CHOLECYSTECTOMIES/CONVERSION TO OPEN SURGERY}

doi:10.1136/gutjnl-2012-302514d.136

P Asaad, ${ }^{*}$ K G Pursnani, R S Date. Upper Gastrointestinal Surgery, Lancashire Teaching Hospitals NHS Foundation Trust, Chorley, UK

Introduction Conversion to open surgery used to be a marker of difficult cholecystectomy. With increasing experience conversion rate has reduceed significantly, but the difficulties remain the same. Both, conversion and difficult cholecystectomy have impact on operation time. The aim of this study is to identify the major predictive factors for "difficult" cholecystectomies, which are either continued laparoscopically or subsequently converted.

Methods A retrospective review of all the consecutive laparoscopic cholecystectomies, performed by a single surgeon, in a district general hospital in the UK, from January to December 2011, was undertaken. Association of intra-operative difficulties or conversion to open surgery, with the following factors was studied-Age, gender, liver function tests, jaundice, cholecystitis, pre-operative ERCP, pancreatitis, and radiological findings.

Results During the study period 180 patients underwent cholecystectomy, of which 10 were converted to open surgery $(5.6 \%)$, while $30(16.6 \%)$ others were deemed "difficult dissections" but the operations were still completed laparoscopically. Previous cholecystitis $(n=45)$ seems to be the most important predictor of difficulty, with $71 \%$ of patients requiring conversion or being considered a "difficult dissection." Another very useful predictor is previous ERCP $(n=14)$, with $64.5 \%$ of these patients being either conversions or "difficult dissections." The conversion rate and difficult laparoscopic dissection rate was $8 \%$ and $24 \%$ respectively for men $(n=37)$ and $5 \%$ and $15 \%$ for women $(n=143)$. Among the patients with previous pancreatitis, none required conversion and $29 \%$ had difficult dissections. The conversion and difficult dissection rates increased with age $(7 \%$ and $4 \%$ for age $<40,20 \%$ and $1 \%$ for age $40-60$, and $20.5 \%$ and $9.5 \%$ for age $>60$ respectively).

Conclusion Bile duct stones managed with pre-operative ERCP, cholecystitis and male gender appear to be the major predictors of difficult cholecystectomies/conversion to open surgery. This ability to predict the difficulty of the procedure might help the surgeon prepare for any technical difficulties that may arise, organise the theatre list more efficiently, and offer the patient more accurate information and counselling prior to the procedure.

Competing interests None declared.

\section{PWE-137 THE ROLE OF ${ }^{99 M}$ TECHNETIUM-LABELLED HEPATO IMINO DIACETIC ACID (HIDA) IN THE MANAGEMENT OF BILIARY PAIN}

doi:10.1136/gutjnl-2012-302514d.137

R Dave, * A Cockbain, G Toogood. Transplant and HPB Surgery, St James University Hospital, Leeds, UK

Introduction Biliary pain is a common presentation in the acute surgical take and the surgical clinic. In patients with normal ultrasound findings, symptoms can sometimes be disregarded as being non-specific. We propose that in this cohort, a HIDA scan is a useful investigation, and patients with a positive test have good results following cholecystectomy.

Methods We obtained reports of all HIDA scans with an abnormal ejection fraction $(\mathrm{EF}<40 \%$ ) performed in our centre from 15 May 2007 to 28 December 2010. This database was cross-linked with a prospectively-maintained database and electronic records of patients undergoing cholecystectomy in the same period. All patients with a positive HIDA who went on to have laparoscopic cholecystectomy (LC) were followed-up by a review of the electronic records, and a telephone interview to asses symptom improvement.

Results 50 patients were investigated. Mean age was 48, and majority were female. Ultrasound findings revealed no stone disease and normal gall bladder in $96 \%$. $92 \%$ of patients were happy with the decision to proceed with LC, and $87.5 \%$ felt that their symptoms were improved (62.5\% "Very Much Improved"). Post-operatively, $56.3 \%$ had no residual pain whatsoever and $31.3 \%$ had only occasional mild discomfort. The histology was pathological in $83 \%$; $29 \%$ had stones in the gall bladder. During the HIDA scan, injection of a CCK-analogue caused pain in $56 \%$. Symptoms were "Very Much Improved" after LC in $74 \%$ and $44 \%$ respectively in the responders and non-responders to CCK-analogue injection. The sensitivity of CCK-analogue injection was $68 \%$ and specificity was $50 \%$

Conclusion HIDA scan is a useful clinical tool in the diagnosis and management of patients with typical biliary pain and normal ultrasound. Outcomes following LC in this cohort of patients are favourable, with high patient satisfaction. The injection of a CCK analogue is a sensitive adjunct to the test, but non-response does not rule out benefit from LC.

Competing interests None declared.

\section{PWE-138 THE INCIDENCE AND MANAGEMENT OF CYSTIC DUCT STONES: THE INTRA-OPERATIVE CHOLANGIOGRAM IS MORE THAN JUST A DIAGNOSTIC TOOL}

doi:10.1136/gutjnl-2012-302514d.138

${ }^{1} \mathrm{R}$ Dave, ${ }^{*} \mathrm{~N}$ Yeomans, ${ }^{1} \mathrm{~A}$ Cockbain, ${ }^{1} \mathrm{G}$ Toogood. ${ }^{1}$ Transplant and HPB Surgery, St James University Hospital, Leeds, UK; ${ }^{2}$ Department of Colorectal Surgery, St James University Hospital, Leeds, UK

Introduction Use of the Intraoperative cholangiogram (IOC) was introduced by Mirizzi in 1931, who recommended its routine use Currently, routine IOC during laparoscopic cholecystectomy (LC) remains a controversial issue. Unsuspected common bile duct (CBD) stones are reported in only between $2 \%$ and $3 \%$ of cases, whereas "post-cholecystectomy syndrome (PCS)," is reported in 10\%-40\%. A potential cause of this is retained stones within the cystic duct (CD) remnant. We aimed to identify the intra-operative incidence of $\mathrm{CD}$ stones and the incidence of post-operative complications following routine IOC.

Methods We analysed a prospectively maintained database of all LC and routine IOC performed by the senior author. Since 7 April 2010, the incidence of CD stones/sludge was prospectively recorded-once the incision on the $\mathrm{CD}$ had been made, the $\mathrm{CD}$ and the $\mathrm{CBD}$ were "milked" in a retrograde fashion to remove any debris prior to introduction of the catheter for IOC. Impacted CD stones were crushed laproscopically. We also analysed the entire database prospectively collected since 1999 for the incidence of CBD stones. T-test (continuous) and $\mathrm{Chi}^{2}$ (categorical) tests were used to analyse predictors of $\mathrm{CD}$ stones.

Results 248 LC with IOC had been recorded from 7 April 2010. In this cohort, the incidence of CD stones was $13 \%(\mathrm{~N}=33 / 248)$ and CD sludge was $6 \%(N=15 / 248)$. The presence of CD stones was not 